\section{ORIGINAL RESEARCH}

S. Fujii

Y. Kanasaki

E. Matsusue

S. Kakite

T. Kminou

T. Ogawa

\title{
Demonstration of Cerebral Venous Variations in the Region of the Third Ventricle on Phase- Sensitive Imaging
}

\begin{abstract}
BACKGROUND AND PURPOSE: Susceptibility-weighted (SW) MR imaging has enabled noninvasive visualization of the cerebral veins and has shed light on the nature of venous architecture. For successful surgery of the third ventricle, understanding of the anatomy of the subependymal veins of the lateral ventricle and their relationships to the foramen of Monro is required preoperatively. The purpose of this study was to evaluate the anatomic variations of the subependymal veins around the third ventricle by use of phase-sensitive imaging (PSI) on the basis of principles similar to those of SW $M R$ imaging.
\end{abstract}

\begin{abstract}
MATERIALS AND METHODS: Included in this study were 642 sides in 321 patients. The courses of the anterior septal vein (ASV), thalamostriate vein, and internal cerebral vein (ICV) were evaluated. We classified these into 4 types (IA, IB, IIA, IIB) on the basis of standard classic angiographic criteria. The classification is based on their relationship with the ASV-ICV junction and the presence of a venous angle or a false venous angle, according to the method in a previous study. Other venous variations were classified as type III.
\end{abstract}

RESULTS: A venous angle was formed in 519 (80.9\%), whereas a false venous angle was formed in $123(19.1 \%)$. The ASV-ICV junction was located at the venous angle (type IA) in 407 (63.4\%) of 642 sides. In 235 sides $(36.6 \%)$, the ASV-ICV junction was located posteriorly beyond the foramen of Monro (types IB, IIA, IIB, and III).

CONCLUSIONS: PSI is useful for understanding normal variations of the subependymal veins in the region of the third ventricle.

S usceptibility-weighted (SW) MR imaging is a 3D gradientecho imaging technique based on the blood oxygen leveldependent (BOLD)-induced phase effects between venous blood and the surrounding brain parenchyma., ${ }^{1,2}$ The technique of SW MR imaging for visualization of the venous system is based on the BOLD effect, which exploits the fact that paramagnetic deoxyhemoglobin causes a local magnetic field inhomogeneity, resulting in both a reduction of $\mathrm{T} 2^{*}$ and a phase difference between the venous vessel and its surroundings. Thus, SW MR imaging enables noninvasive visualization of the cerebral veins and has shed light on the nature of venous architecture $^{3-5}$ and cerebral venous malformations. ${ }^{6-8}$ Currently, SW MR imaging is used mainly to investigate brain parenchymal lesions such as multiple sclerosis, ${ }^{9}$ small hemorrhage, ${ }^{10}$ and tumors. ${ }^{6-8}$

Cerebral angiography initially played the dominant role in the evaluation of cerebral venous anatomy in patients with brain tumors, but its role is now greatly reduced following advances in tomographic imaging. In surgery of the third ventricle, however, successful surgery necessitates understanding of the anatomy of the subependymal veins of the lateral ventricle and their relationships to the foramen of Monro preoperatively. ${ }^{11}$ Therefore, radiologists should be familiar with the cerebral venous anatomy in the region of the third ventricle and have an appreciation of the venous variations in this area.

Received April 9, 2009; accepted after revision May 21.

From the Division of Radiology, Department of Pathophysiological and Therapeutic Science, Faculty of Medicine, Tottori University, Yonago, Japan.

Please address correspondence to Shinya Fujii, Division of Radiology, Department of Pathophysiological and Therapeutic Science, Faculty of Medicine, Tottori University, 36-1, Nishi-cho, Yonago, Tottori 683-8504, Japan; e-mail: sfujii@grape.med.tottori-u.ac.jp DOI 10.3174/ajnr.A1752
Phase-sensitive imaging (PSI), on the basis of principles similar to those of SW MR imaging, is obtained by multiplication of the phase and magnitude images by use of Windowsbased software. ${ }^{12}$ In this study, we demonstrate cerebral venous variations in the region of the third ventricle on PSI.

\section{Materials and Methods}

\section{Patient Population}

Included in this retrospective study were 734 sides in 367 consecutive patients who underwent MR imaging examinations including PSI at our institution between November 2007 and October 2008. PSI images of the brain were obtained for screening of headache or evaluation of hemorrhage, infarction, brain tumor, or degenerative diseases. We excluded 46 sides in 23 patients because the third ventricular area was not included on PSI. Sixteen sides in 8 patients were excluded because of motion artifacts or susceptibility artifacts because of the nasal sinuses or dental prostheses. In addition, 30 sides in 15 patients were excluded because their images could not be evaluated due to susceptibility artifacts caused by an intraventricular or basal ganglion hemorrhage around the third ventricle. A total of 642 sides in 321 consecutive patients (age range, 0-90 years; mean age, 58 years) who underwent PSI on a 3T MR imaging system were enrolled in the study. However, there were no cases of a third ventricular lesion in the present study. The Institutional Review Board approved our study, which did not require informed consent.

\section{MR Imaging Technique}

We obtained all brain MR imaging with a 3T MR system (Signa Excite HD; GE Healthcare, Milwaukee, Wisconsin) using an 8-channel high-resolution brain phased array coil (Invivo Corp., Gainesville, Florida). 


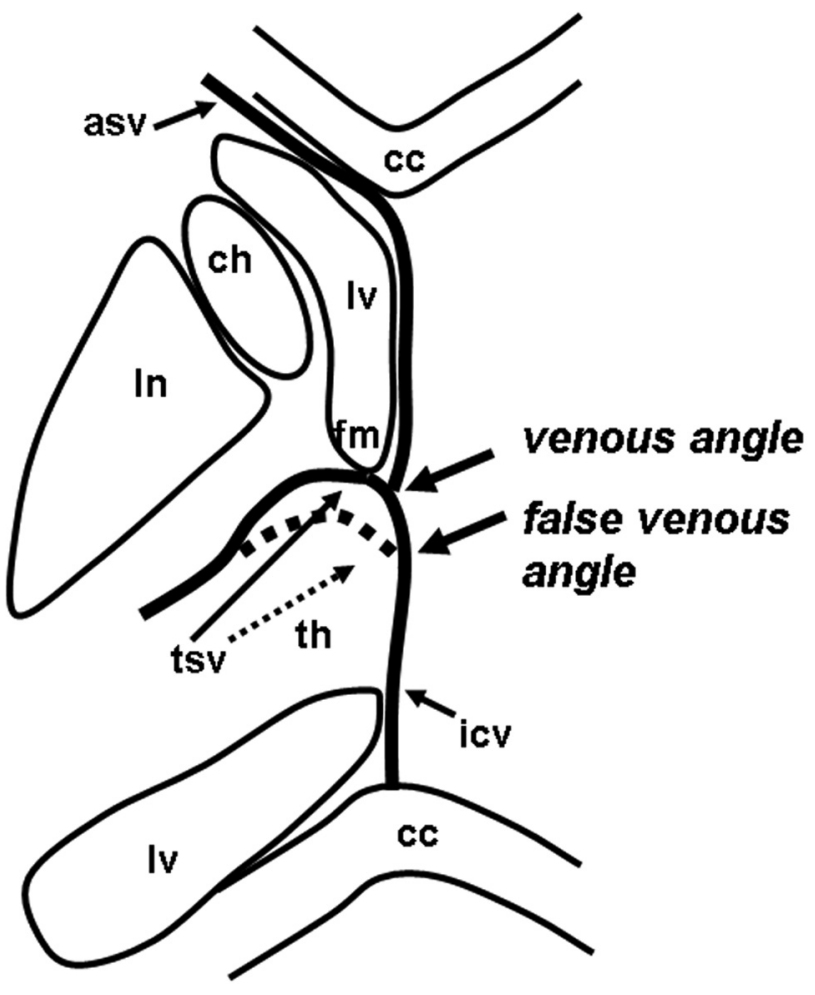

Fig 1. Diagram demonstrating the venous angle and false venous angle (Iv, lateral ventricles; asv, anterior septal vein; tsv, thalamostriate vein; icv, internal cerebral vein; fm, foramen of Monro; cc, corpus callosum; In, lentiform nucleus; ch, head of caudate nuclei; th, thalamus).

We acquired PSI with a 3D echo-spoiled gradient-echo sequence with flow compensation using the following imaging parameters: TR, $45 \mathrm{~ms}$; TE, $30 \mathrm{~ms}$; FA, $20^{\circ}$; FOV, $21 \mathrm{~cm}$; matrix, $512 \times 192$; section thickness, $1.5 \mathrm{~mm}$; acquisition time, $7 \mathrm{~min} 40$ to $50 \mathrm{~s}$. All images were obtained in the axial plane. We postprocessed the phase-sensitive images using a high-pass filter and then con- verted the images into negative phase masks that were multiplied 4 times into the corresponding magnitude images using research software (PSIRecon; GE Yokogawa Medical Systems, Tokyo, Japan). We used a minimal-intensity projection (PSI) to display the processed data and contiguous $10.5-\mathrm{mm}$-thick sections with $7-\mathrm{mm}$ overlap in the transverse plane (Advantage workstation version 4.1; GE Healthcare).

\section{Evaluation of the Venous Anatomy in the Region of the Third Ventricle}

Two experienced neuroradiologists consensually assessed each side with regard to venous variation on PSI. The reviewers identified the anterior septal vein (ASV) and thalamostriate vein (TSV) as running the following courses. The ASV initially runs posteromedially on the anterior wall of the frontal horn and behind the genu of the corpus callosum. When it reaches the anteromedial corner of the frontal horn, it runs backwards along the septum pellucidum and continues backward along its lower border. It then follows the lateral border of the anterior column of the fornix, generally joining the internal cerebral vein (ICV) at the posterosuperior margin of the foramen of Monro. ${ }^{13}$

The TSV begins in the region of the anterior wall of the atrium or in the inferolateral wall of the body of the lateral ventricle, in the linea terminalis. It passes anteriorly and medially, beneath the stria terminalis, receiving several transverse caudate veins into which the longitudinal caudate veins drain, on its way toward the foramen of Monro. At the level of the foramen of Monro, the TSV runs around the anterior tubercle of the thalamus and receives the vein from the head of the caudate nucleus. It then passes medially, generally joining the ICV at the posterosuperior margin of the foramen of Monro. ${ }^{13}$

The characteristic U-shaped junction of the TSV and ICV adjacent to the posterior margin of the foramen of Monro is referred to as the venous angle (Fig 1). When the junction of the TSV and ICV lies beyond the posterior margin of the foramen of Monro, this variation

\section{Type IA}

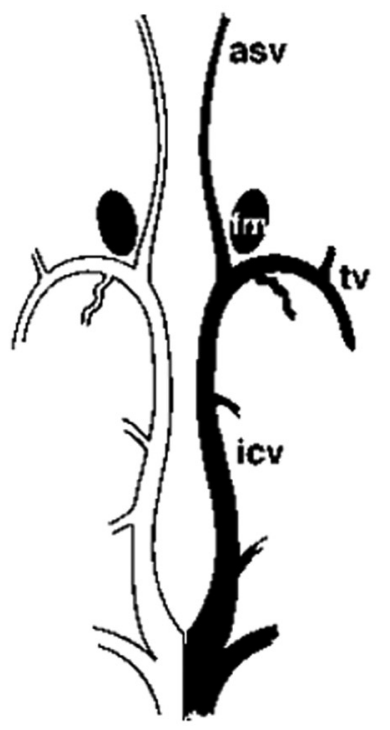

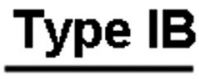

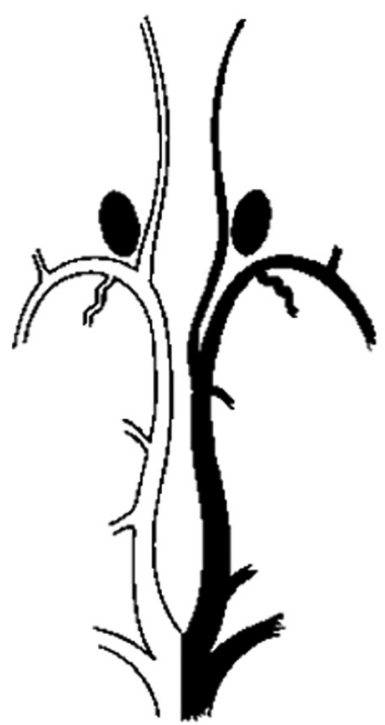

Type IIA

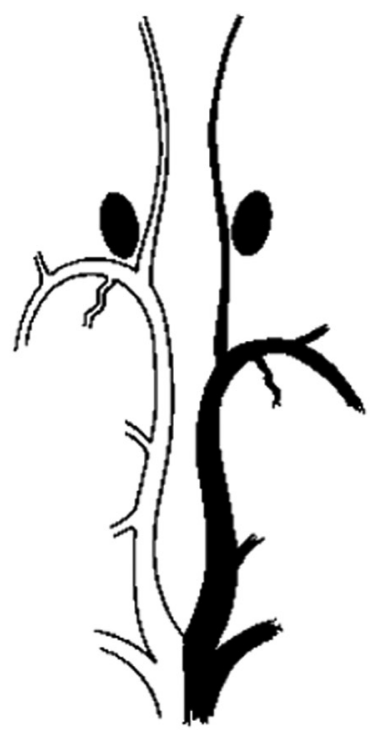

Type IIB

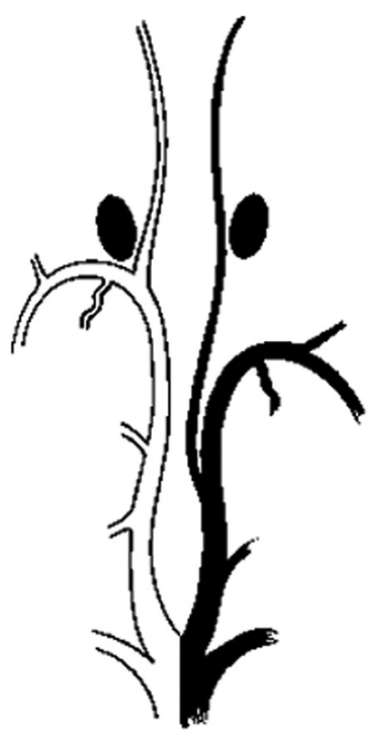

Fig 2. Variations in location of the ASV-ICV junction relative to the foramen of Monro (fm). (Reprinted by permission of Ture U, Yasargil, MG, Al-Mefty 0. The transcallosal-transforaminal approach to the third ventricle with regard to the venous variations in this region. J Neurosurg 1997;87:706-1511). 

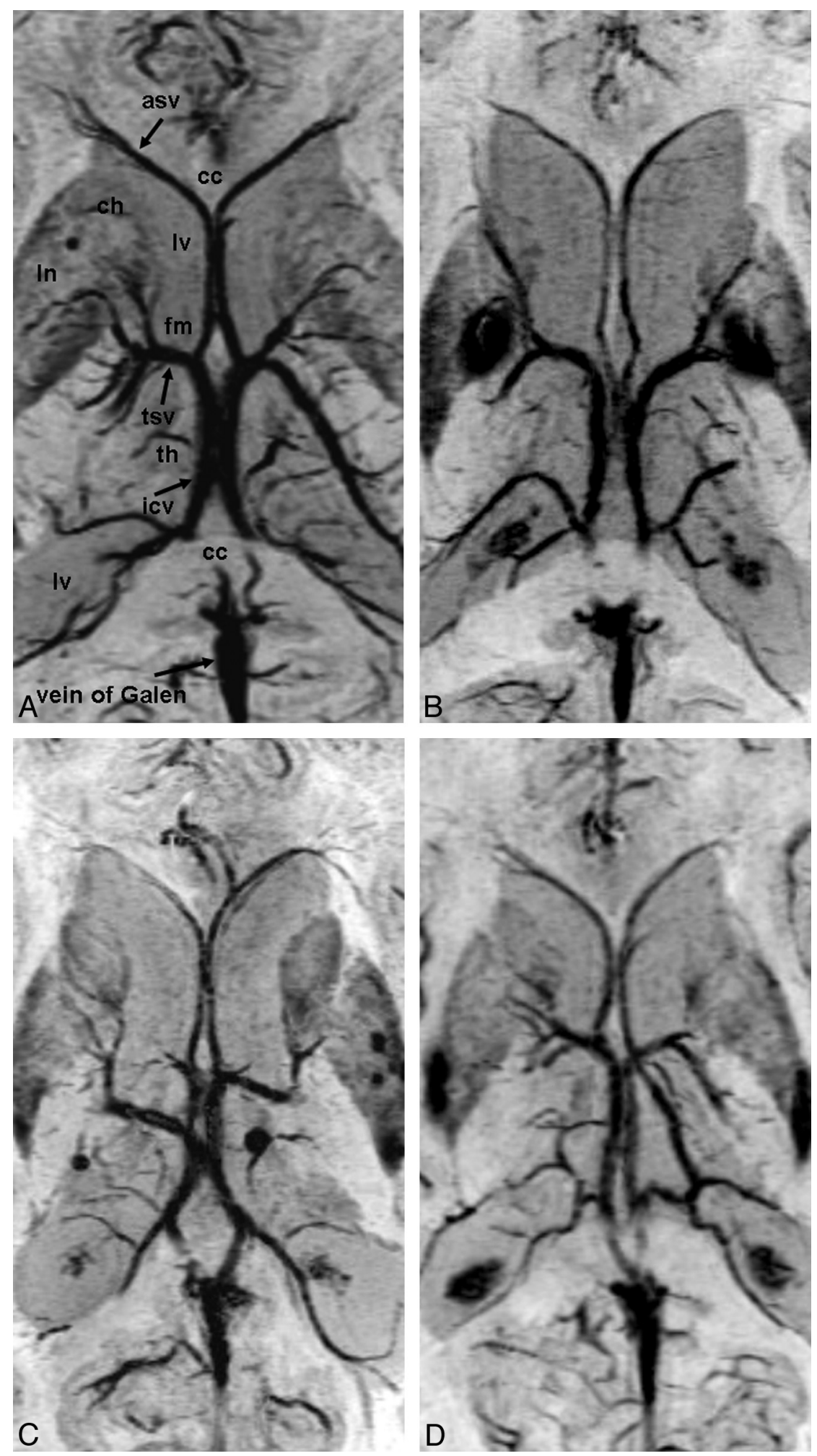

Fig 3. Cerebral venous variations in the region of the third ventricle on PSI. Abbreviations are the same as in Fig 1. A, Type IA: the ASV-ICV junction is located at the venous angle, which lies adjacent to the posterior margin of the foramen of Monro. B, Type IIA (left): the ASV joined the false venous angle, which lies beyond the foramen of Monro. Type IB (right): the ASV joined the mainstem of the ICV posteriorly beyond both the foramen of Monro and the venous angle. C, Type IA (left), type IIB (right): the ASV joined the mainstem of the ICV far beyond both the foramen of Monro and the false venous angle. D, Type III (left): the ASV joined the medial atrial vein and the venous angle is formed. Type IA (right).

is referred to as the false venous angle (Fig 1). We classified these into 4 types on the basis of their relationship with the ASV-ICV junction and the presence of a venous angle or a false venous angle, according to the method used in a previous study (Fig 2). ${ }^{11}$

The 4 types were classified as follows:
- Type IA: ASV-ICV junction was located at the venous angle, which lies adjacent to the posterior margin of the foramen of Monro. In the other types, the ASV-ICV junction was located beyond the posterior margin of the foramen of Monro, in 3 different locations. 
- Type IB: the ASV joined the mainstem of the ICV posteriorly beyond both the foramen of Monro and the venous angle.

- Type IIA: the ASV joined the false venous angle, which lies beyond the foramen of Monro.

- Type IIB: the ASV joined the mainstem of the ICV far beyond both the foramen of Monro and the false venous angle. We classified any other venous variations as type III.

\section{Results}

Figure 3 shows representative cases of the 4 types of cerebral venous variation in the region of the third ventricle. Data regarding the types of cerebral venous variation in the region of the third ventricle are summarized in the accompanying $\mathrm{Ta}$ ble. In only 1 case of type III, the ASV joined the medial atrial vein (Fig 3).

In $519(80.9 \%)$ of 642 sides, the venous angle (TSV joins the ICV at the margin of the foramen of Monro) was formed. In $123(19.1 \%)$ of 642 sides, a false venous angle was formed (Table; Fig 3).

In $407(63.4 \%)$ of 642 sides, the ASV-ICV junction was located at the venous angle (type IA). In 235 (36.6\%) of 642 sides, the ASV-ICV junction was located posteriorly, beyond the foramen of Monro (types IB, IIA, IIB, and III) (Fig 3).

\section{Discussion}

Surgery in the region of the third ventricle poses a considerable challenge, even for experienced neurosurgeons. The numerous publications describing various surgical approaches attest to the difficulties encountered when exploring this area. ${ }^{11}$ With use of the transcortical-transventricular and the anterior transcallosal approaches, access is gained to the anterior and middle portions of the third ventricle. The anterior transcallosal approach usually provides a direct and adequate pathway to the lateral ventricles, where the foramen of Monro serves as a natural entrance into the anterior and middle portions of the third ventricle, particularly when the foramen is dilated by tumor tissue. When the foramen of Monro is not dilated by a tumor, however, access to the midsuperior portion of the third ventricle is limited. The location of the ASV-ICV junction relative to the foramen of Monro is the key to this exposure. When the ASV-ICV junction is located posteriorly, beyond the foramen of Monro, the transforaminal exposure can be adapted. The foramen of Monro, ipsilateral to the more posteriorly located ASV-ICV junction, can be enlarged along the choroidal fissure as far as the junction to provide a wide access to the third ventricle. ${ }^{11}$ In our study, PSI demonstrated cerebral venous variations in the region of the third ventricle. Therefore, PSI may play an important role in the preoperative planning for lesions located around the third ventricle.

Various studies describe the deep venous system of the brain and venous variations in the region of the foramen of Monro. ${ }^{14-24}$ On cerebral angiography, the incidence of a false venous angle is reported to vary between $14 \%$ and $26 \%{ }^{16-18,20-22}$ In our study, we observed the presence of a false venous angle in $19.1 \%$ of sides, which is within the range reported for angiography.

According to an anatomic study, ${ }^{11}$ the frequency of a false venous angle (beyond the foramen of Monro) was 32.5\%, and the posterior location of the ASV-ICV junction was $47.5 \%$. The frequencies reported in the present study are low com-

\begin{tabular}{l}
\hline $\begin{array}{l}\text { Frequency of each type of cerebral venous variation in the region } \\
\text { of the third ventricle }\end{array}$ \\
\begin{tabular}{lrrr}
\hline \multicolumn{2}{l}{ Type } & ASV-ICV Junction & Type of Venous Angle \\
\hline IA & $407(63.4 \%)$ & Foramen of Monro & Venous angle \\
IB & $111(17.3 \%)$ & Beyond foramen of Monro & Venous angle \\
IIA & $106(16.5 \%)$ & Beyond foramen of Monro & False venous angle \\
IIB & $17(2.6 \%)$ & Beyond foramen of Monro & False venous angle \\
III & $1(0.2 \%)$ & Beyond foramen of Monro & Venous angle \\
\hline
\end{tabular}
\end{tabular}

Note:-ASV-ICV indicates anterior septal vein-internal cerebral vein.

pared with this anatomic study. The reason for this discrepancy is not clear but may relate to the small population (40 hemispheres) in the anatomic study and the cases in the present PSI study for which accurate assessment was difficult because of the very short distance between the venous junction and the foramen of Monro.

MR venography is used in the evaluation of the venous anatomy ${ }^{24}$; however, we believe that SW MR imaging, including PSI, should be obtained for evaluation of the deep cerebral venous anatomy because it can depict the cerebral vein with high resolution, ${ }^{3-5}$ and it enables evaluation of brain parenchymal lesions, particularly a small hemorrhage. ${ }^{10}$ Therefore, we consider PSI to be a useful MR imaging technique that could be added to imaging protocols.

There were some limitations in our study. First, the potential existed for artifacts in PSI. Application of this imaging method may be difficult in areas with $\mathrm{B}_{0}$ inhomogeneity, restricting its use to anatomic brain regions free of large static field inhomogeneities caused by susceptibility differences, such as regions near the paranasal sinuses and skull base. ${ }^{5}$ Susceptibility artifacts because of hemorrhage are naturally stronger on PSI than that for other imaging sequences. Therefore, some patients were excluded from our study, but the proportion was low.

Second, the sequence parameters were optimized for evaluation of intraparenchymal lesions rather than for visualization of veins. Although we could evaluate the veins in most cases, further examination may be needed.

Third, surgical confirmation was not performed because third ventricular lesions were not included because of the absence of such a case. A prospective study is necessary for sideby-side comparison of the MR imaging results with the surgical findings.

\section{Conclusions}

PSI is useful for gaining an understanding of normal variations of the subependymal veins in the region of the third ventricle. PSI may play an important role in the preoperative planning of lesions in the third ventricle.

\section{Acknowledgments}

We thank Eijirou Yamashita, BS; Takuro Tanaka, BS; Naoki Iwata, BS; and Shota Sakimoto, BS, for technical support in obtaining the high-quality MR images used in this study.

\section{References}

1. Reichenbach JR, Haacke EM. High-resolution BOLD venographic imaging: a window into brain function. NMR Biomed 2001;14:453-67

2. Rauscher A, Sedlacik J, Barth M, et al. Nonnvasive assessment of vascular architecture and function during modulated blood oxygenation using suscep- 
tibility weighted magnetic resonance imaging. Magn Reson Med 2005;54: 87-95

3. Reichenbach JR, Venkatesan R, Schillinger DJ, et al. Small vessels in the human brain: MR venography with deoxyhemoglobin as an intrinsic contrast agent. Radiology 1997;204:272-77

4. Lee BC, Vo KD, Kido DK, et al. MR high-resolution blood oxygenation leveldependent venography of occult (low-flow) vascular lesions. AJNR Am J Neuroradiol 1999;20:1239-42

5. Reichenbach JR, Barth M, Haacke EM, et al. High-resolution MR venography at 3.0 Tesla. J Comput Assist Tomogr 2000;24:949-57

6. Sehgal V, Delproposto Z, Haacke EM, et al. Clinical applications of neuroimaging with susceptibility-weighted imaging. J Magn Reson Imaging 2005;22:439-50

7. Thomas B, Somasundaram S, Thamburaj K, et al. Clinical applications of susceptibility weighted MR imaging of the brain - a pictorial review. Neuroradiology 2008;50:105-16

8. Tong KA, Ashwal S, Obenaus A, et al. Susceptibility-weighted MR imaging: a review of clinical applications in children. AJNR Am J Neuroradiol 2008;29: $9-17$

9. Tan IL, van Schijndel RA, Pouwels PJ, et al. MR venography of multiple sclerosis. AJNR Am J Neuroradiol 2000;21:1039-42

10. Akter M, Hirai T, Hiai Y, et al. Detection of hemorrhagic hypointense foci in the brain on susceptibility-weighted imaging clinical and phantom studies. Acad Radiol 2007;14:1011-19

11. Ture U, Yasargil MG, Al-Mefty O. The transcallosal-transforaminal approach to the third ventricle with regard to the venous variations in this region. J Neurosurg 1997;87:706-15
12. Kakeda S, Korogi Y, Kamada K, et al. Signal intensity of the motor cortex on phase-weighted imaging at 3T. AJNR Am J Neuroradiol 2008;29:1171-75

13. Huang YP. Deep cerebral veins. In: Salamon G, Huang YP, eds. Radiologic Anatomy of the Brain. Berlin: Springer-Verlag; 1976:210-61

14. Johanson $C$. The central veins and deep dural sinuses of the brain; an anatomical and angiographic study. Acta Radiol Suppl 1954;107:1-184

15. Krayenbuhl HA, Yasargil MG. Cerebral Angiography, 2nd ed. Philadelphia: Lippincott; 1968

16. Lang J. Surgical anatomy of the hypothalamus. Acta Neurochir 1985;75:5-22

17. Mokrohisky JF, Paul RE, Lin PM, et al. The diagnostic importance of normal variants in deep cerebral phlebography. With special emphasis on the true and false "venous angles of the brain" and evaluation of venous angle measurement. Radiology 1956;67:34-47

18. Ono M, Rhoton AL Jr, Peace D, et al. Microsurgical anatomy of the deep venous system of the brain. Neurosurgery 1984;15:621-57

19. Probst FP. Position of the "venous angle" in the median sagittal plane. A new topometric method. Acta Radiol (Diagn) 1970;10:271-88

20. Ring BA. Variations in the striate and other cerebral veins affecting measurements of the "venous angle". Acta Radiol 1959;52:433-47

21. Stein RL, Rosenbaum AE. Deep supratentorial veins. Section I. Normal deep cerebral venous system. In: Newton TH, Potts DG, eds. Radiology of the Skull and Brain. Angiography. Vol 2. St. Louis: Mosby;1974:1903-98

22. Wolf BS, Huang YP. The subependymal veins of the lateral ventricles. $A J R$ Am J Roentgenol 1964;91:406-26

23. Zimmer AE, Annes GP. The septal vein. An indicator of space occupying lesions in the anterior cranial fossa. Radiology 1966;87:813-23

24. Cimsit NC, Ture U, Ekinci G, et al. Venous variations in the region of the third ventricle: the role of MR venography. Neuroradiology 2003;45:900-04 